\title{
Analysis and design of a WLAN OFDM transmitter with digital filters
}

\author{
Pavlos Kolovos \\ Department of Electrical and \\ Computer Engineering, \\ University of Patras, Greece \\ kolobos@ceid.upatras.gr
}

\author{
Eleni Fotopoulou \\ Department of Electrical and \\ Computer Engineering, \\ University of Patras, Greece \\ helenf@ece.upatras.gr
}

\author{
Thanos Stouraitis \\ Department of Electrical and \\ Computer Engineering, \\ University of Patras, Greece \\ thanos@ece.upatras.gr
}

\begin{abstract}
This paper introduces a study and an implementation of filters in the digital part of an Orthogonal Frequency Division Multiplexing (OFDM) based transmitter. An efficient computational technique is presented for design with interpolation filters and discusses the corresponding VLSI architecture issues. By exploiting the redundancy into the cyclic prefix part of the OFDM symbol, the computational load of the transmitter is reduced by approximately $20 \%$ for the IEEE 802.11 WLAN standard.
\end{abstract}

\section{Keywords}

OFDM, WLAN, digital filters, VLSI architecture

\section{INTRODUCTION}

The current increase of demand for wireless communications applications, dictates a necessity for high-speed and spectral-efficient communications over wireless highly dispersive channels. Orthogonal Frequency Division Multiplexing (OFDM) has been selected as an efficient solution for high-speed digital wireless communications and is employed in current wireless communication standards, such as IEEE $802.11 \mathrm{~b}$ in the $5 \mathrm{Ghz}$ band and $802.11 \mathrm{~g}$ in the $2.4 \mathrm{Ghz}$ band, for wireless LANs, DVB for digital terrestrial TV broadcasting, WiMax for wireless MANs, 802.15 for wireless PANs etc.

In the 802.11 WLAN standard [2] the baseband signal is generated by using the IFFT, then a guard interval (also called a cyclic-prefix-CP) is added to make the system robust to multipath. The IFFT modulation produces a spectra of overlapping subcarriers, where each subcarrier is orthogonal to all other subcarriers. Respectively, the transmitted data are demodulated by an FFT in the receiver.

${ }^{*}$ We thank the European Social Fund (ESF), Operational Program for Educational and Vocational Training II (EPEAEK II)

Permission to make digital or hard copies of all or part of this work for personal or classroom use is granted without fee provided that copies are not made or distributed for profit or commercial advantage and that copies bear this notice and the full citation on the first page. To copy otherwise, to republish, to post on servers or to redistribute to lists, requires prior specific permission and/or a fee. MOBIMEDIA 2007, August 27-29, Nafpaktos, Greece Copyright (C) 2007 ICST 978-963-06-2670-5 DOI 10.4108/ICST.MOBIMEDIA2007.1814
In this paper, the study and implementation of filters in the digital part of an OFDM transmitter is presented. We investigate the digital filters that need to be applied at the digital part of the transmitter and propose an architecture where the filtered cyclic prefix part is not explicitly computed; instead it is constructed from the filtering of the useful OFDM symbol part. In this way the proposed algorithm, avoids the filtering of the total OFDM symbol, thus significantly reducing the number of multiplications needed by approximately $20 \%$, for the case of a 64 -point IFFT with cyclic prefix of one fourth of the OFDM symbol.

In previous work upsampling with interpolation filters has been discussed. A technique called circular time domain interpolation is applied where the 64-point IFFT result is both pre- and post- extended and then upsampled and filtered requiring additional computations [6]. Construction of the CP after filtering has already been presented in [4]. It is based on a combination of a radix-4 Multipath-Delay Commutator FFT architecture [5] with 4 parallel filters. In this paper we propose an architecture which outperform the previous designs in terms of area, number of computations, and utilization.

The remainder of the paper is organized as follows: In Section 2, a brief overview of the OFDM transmitter is and the analysis for the required digital filters is presented. Section 3 describes the proposed architecture. Results of implementation are discussed in Section 4 and conclusions are given in Section 5.

\section{DIGITAL FILTERING IN OFDM TRANS- MITTERS}

A brief description of the organization of an OFDM transmitter [8], follows. The binary input data are encoded by a forward error correction scheme. The encoded data are subsequently interleaved and mapped onto QAM values. After the pilot tones are inserted, the IFFT modulation is performed to produce the useful OFDM symbol part.

The subcarrier orthogonality in OFDM is retained, when the signal is transmitted over a non-dispersive channel. To deal with the time dispersion, a guard interval is introduced between successive OFDM symbols. In most cases, the guard interval is a cyclic repetition of a number of the last samples of the IFFT output, pre-pended to the symbol part, called the cyclic prefix (CP). Due to bandwidth restrictions the $\mathrm{CP}$ is usually limited to less then one quarter of the useful OFDM symbol part. The OFDM symbol, after filtering, is sent to the Digital-to-Analog converter and passes on to the RF part of the modem. 


\subsection{Analog part of an OFDM system}

The baseband complex OFDM signal from the digital part of the transmitter is transmitted using carrier $f_{c}$ and is given by

$$
r_{(R F)}(t)=\operatorname{Re}\left\{r(t) \exp \left(j 2 \pi f_{c} t\right)\right\}
$$

where $R e($.$) represents the real part of the complex variable.$

The spectrum of the transmitted signal must be compliant with the specifications that are given by the 802.11 standard [2] and are shown in Fig. 1

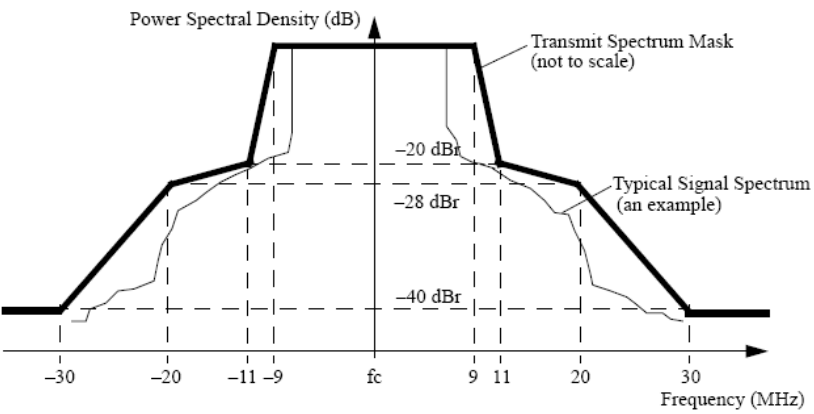

Figure 1: Spectrum of the transmitted signal as defined from the 802.11a standard

According to the standard specifications the number of sub-carriers should be $N=64$. If a $N$-point IFFT is used, bandwidth is pretty narrow and the low pass reconstruction filter applied at the receivers end is characterized by very sudden transitions between passband and stopband frequency areas, which increases implementation complexity of the filter. For this reason and to avoid signal aliasing, the sub-carriers produced by the IFFT procedure should be upsampled. This can be achieved by using a double-in-size IFFT, but this enlarges the required area, requires faster DA converters, and does not achieve reduction of complexity in the analog filter. Thus, there are advantages in the use of interpolation filters after the IFFT [3].

Fig. 2 the block diagram of an OFDM transmitter with interpolation filters is given:

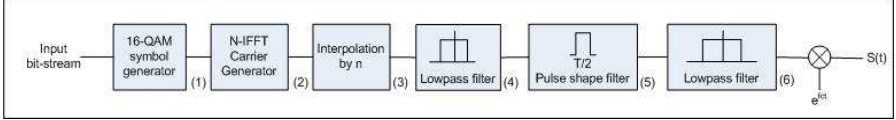

Figure 2: Block diagram of an OFDM transmitter with upsampled subcarriers.

\subsection{Selection of the interpolation filter}

In order to determine the optimal interpolation filter, simulations were performed. We compared the performance of the system in terms of BER and Peak-to-average power ratio (PAPR) to compare filters for different upsampling factors, namely by 2, 4 and 8 . The filters examined were FIR, Chebyshev-I, Butterworth and Elliptic, the length of which differ depending on the upsampling factor.

The simulations include comparison between a system with a double-size IFFT $(N=128)$ and a structure with 64 -point IFFT and interpolation filters.
From the simulation results it is concluded that the minimum BER is accomplished by applying upsampling by 2 . Additionally minimum PAPR is accomplished for FIR and Butterworth filters with upsampling factor 2 and Chebyshev and Elliptic filters for upsampling factor 4. For each of the above groups of filters we ran simulations to determine the optimal system performance in terms of BER and PAPR respectively.

The simulation results are shown in the figures below. In Fig. 3 the results of measuring the PAPR for the two groups of filters are shown, while in Fig. 4 a comparison in terms of BER of the group of interpolation filters with upsampling factor 2 is shown and in Fig. 5 a comparison in terms of $\mathrm{BER}$ of the group of interpolation filters with lowest PAPR is shown.

\begin{tabular}{|c|c|}
\hline MINIMUM BER RESULTS & \\
\hline BEFORE AND AFTER APPLYING THE INTERPC & LATION FILTER \\
\hline PAPR 2 - IFFT & \\
\hline PAPR 2 -FIR(15) INTERPOLATION & $6.693758-7.503788$ \\
\hline PAPR 2 -BUTTERWORTH (17) INTERPOLATION & $6.693758-7.466733$ \\
\hline PAPR 2 -CHEBYSHEV (8) INTERPOLATION & $6.693758-8.214316$ \\
\hline PAPR 2-ELLIPTIC (5) INTERPOLATION & $6.693758-8.285159$ \\
\hline MINIMUM PAPR RESULTS & \\
\hline BEFORE AND AFTER APPLYING THE INTERPC & LATION FILTER \\
\hline PAPR 2-IFFT & 7.637050 \\
\hline PAPR 2-FIR(15) INTERPOLATION & $6.889766-7.683525$ \\
\hline PAPR 2 -BUTTERWORTH (17) INTERPOLATION & $6.889766-7.680486$ \\
\hline PAPR 4-CHEBYSHEV (8) INTERPOLATION & $6.889766-8.391072$ \\
\hline PAPR 4 -ELLIPTIC (5) INTERPOLATION & $6.889766-8.524840$ \\
\hline
\end{tabular}

Figure 3: Comparison of PAPR for the selected interpolation filters

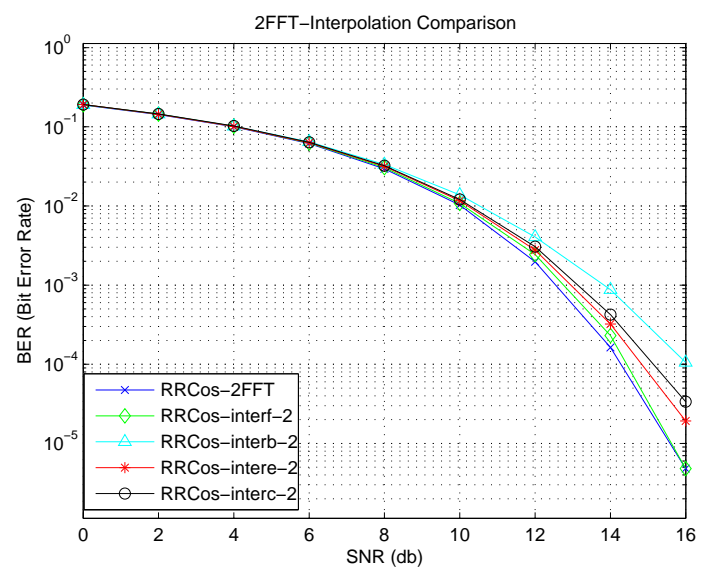

Figure 4: Comparison of BER performance of the iterpolation filters with minimum BER

From the simulation results it can be concluded that lowest BER for the first group of filters is given by a FIR interpolation filter with upsampling factor 2, while in the second group of filters with the lowest PAPR, the Elliptic filter that upsamples by 4 presents the lowest BER but also the highest PAPR (in comparison with the other filters in its group). In contrast, satisfactory results are given by the Butterworth-2 filter in terms of BER and PAPR.

If the selection factor for the interpolation filter is BER 


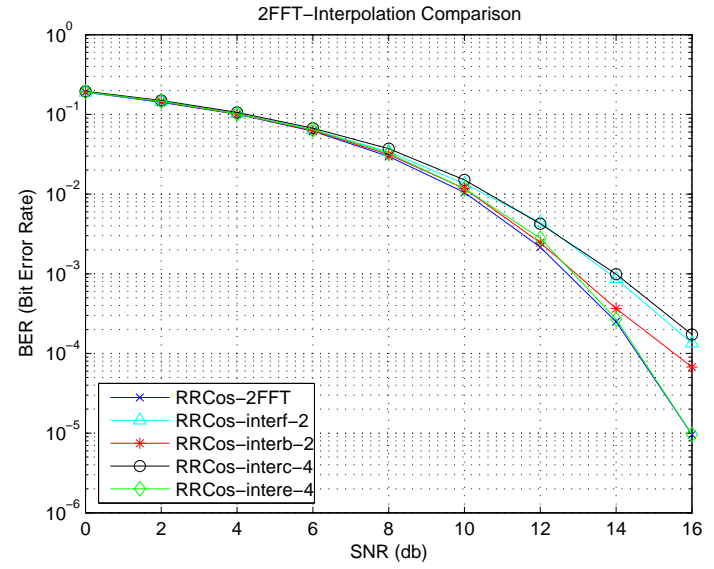

Figure 5: Comparison of BER performance of the iterpolation filters with minimum PAPR

performance then the FIR filter with upsampling factor 2 is the most suitable for implementation. FIR filters are very simple and flexible to implement, while the main advantage is the phase linearity, which is essential for an OFDM system in order to prevent PAPR increase (all circuits between modulator and demodulator have to produce as much as possible linear phase [1]). Concluding the analysis the FIR interpolation filter with upsampling ratio 2 is chosen for implementation.

\subsection{Selection of the low-pass filter}

In this section we determine the optimal digital low pass filter for the implementation. We examine four basic digital low-pass filters Butterworth, Chebyshev I, Elliptical and Root Raised Cosine. The selection of their characteristics (order, cutoff frequency, peak to peak ripple, roll off factor) has been done based on the required spectral mask of Fig. 1.

In Fig. 6 and Fig. 7 comparison of the four low pass filters applied to an upsampled signal of a double-sized IFFT in terms of PAPR and BER respectively is presented.

\begin{tabular}{|lll|}
\hline BEFORE AND AFTER APPLYING THE LOWPASS & FILTER \\
& & \\
PAPR ROot Raised COSine (121) & 2-IFFT & $7.743857-8.458349$ \\
PAPR BUTTERWORTH (6) 2 -IFFT & $7.743857-8.273509$ \\
PAPR CHEBYSHEV (4) 2-IFFT & $7.743857-8.790591$ \\
PAPR ELLIPTIC(3) 2-IFFT & 2-IFT & $7.743857-8.248007$ \\
\hline
\end{tabular}

Figure 6: Comparison of low pass filters in terms of PAPR

Comparing the above simulation results, the optimal lowpass filter with the minimum BER is the Root Raised Cosine(RRC). Considering though the length of the filter which is 121 taps,it is unsuitable for implementation, especially on FPGA where the available resources are limited. Overall the Elliptic filter is more suitable as it presents satisfactory results in BER performance, introduces the lowest PAPR and is small in size-only 3 taps.

To conclude from the analysis and simulation results above for an OFDM system based on the 802.11 specifications with 16-QAM modulation and coding rate $3 / 4$, is consisted of the 64-point IFFT block and an FIR interpolation filter with

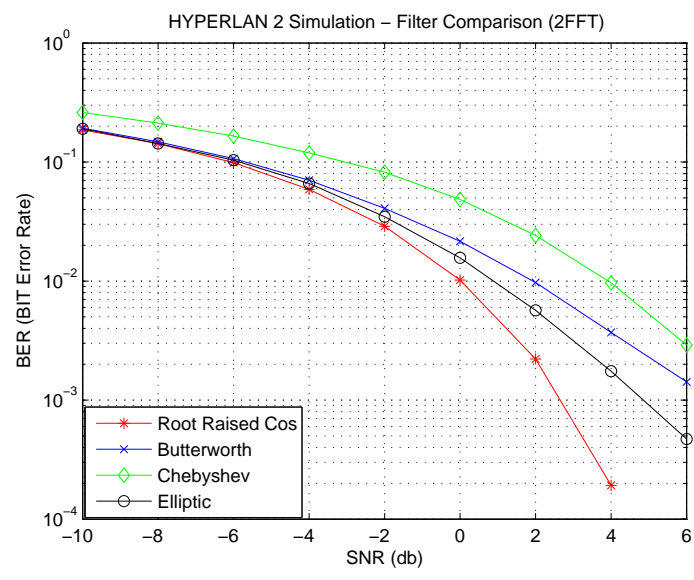

Figure 7: Comparison of low pass filters in terms of BER

15-taps. Finally the produced oversampled signal, passes through an Elliptic low-pass filter with 3 taps, and then is sent to the DAC.

\section{THE PROPOSED ARCHITECTURE}

In this paper, an architecture is proposed to construct the final OFDM symbol. This scheme differs from the straightforward technique, regarding the creation of the cyclic prefix part, as the extension by $N / 4$ of the input data is not necessary. In contrary, the input data sequence remains the same and the convolution of the first $N / 4$ samples is used to compute the cyclic suffix needed to construct the final OFDM symbol. The method used to compute the convolution with the FIR filter is based on the overlap-add method ( [7]), in which the input sequence is divided in sub-blocks and each sub-block is convolved with the filter coefficients. The final filtered output is produced by adding the corresponding appropriate output samples.

The data coming from the IFFT output are in normal order: $x(0), x(1), \ldots, x(63)$

To apply the upsampling filter, zeros are interpolated between the samples.

As defined in the standard, the $N / 4$ first samples of that sequence is used to calculate the cyclic suffix. Thus, using the overlap-add method, the input sequence $x(n)$ can be represented as $x(n)=\sum_{k=0}^{1} x_{k}(n)$, where

$$
\begin{gathered}
x_{0}(n)=\left\{\begin{array}{cc}
x(n), & 0 \leq n \leq N / 4-1 \\
0, & \text { otherwise }
\end{array},\right. \\
x_{1}(n)=\left\{\begin{array}{cc}
x(n), & N / 4-1 \leq n \leq N-1 \\
0, & \text { otherwise }
\end{array},\right.
\end{gathered}
$$

The linear convolution $y(n)$ of the sequence $x(n)$ is then $y(n)=x(n) * h(n)=\left(\sum_{k=0}^{1} x_{k}(n)\right) * h(n)=x_{0}(n) * h(n)+$ $x_{1}(n) * h(n)$

If the impulse response of the filter is $P$ samples and the input sequence is $N$ samples (in this case $N=128$ ), then the convolution output will be $N+P-1$. Thus there is a region of $P-1$ samples over which the first convolution overlaps with the second convolution and the corresponding appropriate output samples must be added resulting in 
$y(n), \quad n=0 \ldots N+P-1$. The convolution $y_{0}(n)$ of the first sequence $x_{0}(n)$ is actually the convolution of the cyclic suffix and may be used to construct the filtered OFDM symbol $y_{\text {OFDM }}$, by adding the appropriate overlapping samples of the sequence generated by $y(n)$.

The construction of the filtered OFDM symbol $y_{\text {OFDM }}$ is given by

$y_{\text {OFDM }}(n)= \begin{cases}y(n), & 0 \leq n \leq N-1 \\ y(n-N)+y_{0}(n-N), & N \leq n \leq P+N-1 \\ y_{0}(n-N), & P+N \leq n \leq P+(N+N / 4)-1\end{cases}$

The computational complexity of the above method is $P N$ multiplications and $(N+2)(P-1)$ additions.

In the straightforward approach, the input sequence to the filter is extended by $N / 4$ and the computational load is $P(N+N / 4)$ multiplications and $(P-1)(N+N / 4)$ additions.

Thus, the gain in computational complexity is $\frac{P(N+N / 4)-P N}{P(N+N / 4)}=$ $20 \%$, for multiplications, and $\frac{(P-1)(N+N / 4)-(N+2)(P-1)}{(P-1)(N+N / 4)}=$ $\frac{N-8}{5 N}$ for additions. For $N=128$, in this implementation, the gain for additions is also $20 \%$.

Fig. 8 shows the proposed architecture for implementing the above method.

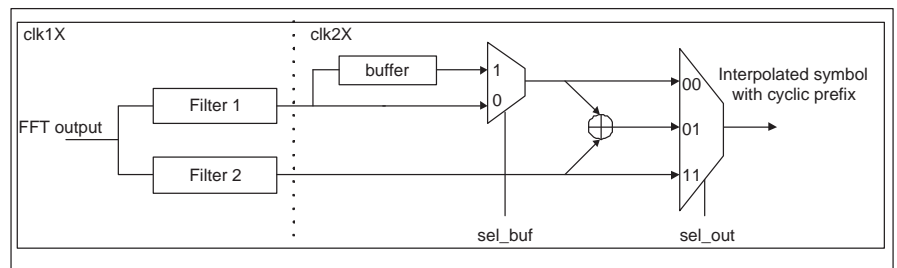

Figure 8: Architecture for computing a filtered OFDM symbol

The output data from the IFFT block, appear in normal order, are sampled by clock $c l k 1 X$, and are fed as inputs to Filters 1 and 2 of Fig 8 . In contrary, the filter outputs are sampled by clock $c l k 2 X$ that operates in double frequency of $c l k 1 X$. All references to clock cycles from this point forward are in correspondence to $c l k 2 X$. The control signals sel_buf and sel_out determine the input and output of the adder, respectively. Filter 1 is used to compute the convolution of sequence $x_{0}, \ldots, x_{15}$, while Filter 2 is used for the sequence $x_{16}, \ldots, x_{63}$. The description and timing for the 1 st method are summarized in Table 1.

\section{IMPLEMENTATION}

Synthesis of the proposed architecture was realized with the Xilinx ${ }^{\mathrm{TM}}$ Ise 5.2i tool and the FPGA device that the design was targeted on was Virtex XCV300 package 352g and speed grade -6 . The results of synthesis for the architecture and the IFFT block are summarized in Table 2.

\section{CONCLUSIONS}

In this paper the analysis and design of interpolation and low pass filters in the digital part of the OFDM transmitter was presented. We introduced an architecture of a 64point FFT architecture followed by digital interpolation filters. By taking advantage of the cyclic repetition of the guard interval of the OFDM symbol, a significant reduction
Table 1: Timing and operation of 1st proposed architecture

\begin{tabular}{|c|c|}
\hline Number of cycles & Description \\
\hline $1-32$ & $\begin{array}{l}\text { Filter } 1 \text { is activated, output data are stored } \\
\text { in a } 46 \text {-word buffer, while the same ele- } \\
\text { ments constitute the output of the system } \\
(\text { sel } \backslash \text { buf }=0 \text { and sel_out }==00)\end{array}$ \\
\hline $33-46$ & $\begin{array}{l}\text { Filter } 2 \text { is also activated (the two filters } \\
\text { are working in parallel), while the out- } \\
\text { put consists of the sum of the outputs of } \\
\text { the first and second filter (overlap in time } \\
\text { (sel\_buf=0 and sel_out==01). After cy- } \\
\text { cle } 46 \text {, Filter } 1 \text { is deactivated. }\end{array}$ \\
\hline $47-96$ & $\begin{array}{l}\text { The output of the system is the output of } \\
\text { Filter } 2\end{array}$ \\
\hline $97-110$ & $\begin{array}{l}\text { The output of the buffer is added to } \\
\text { the output of filter2 ( } \text { sel \_buf=1 and } \\
\text { sel_out==01 ) }\end{array}$ \\
\hline $111-174$ & $\begin{array}{l}\text { The output of the system is provided by } \\
\text { the output of the buffer. }\end{array}$ \\
\hline
\end{tabular}

Table 2: FPGA resource use and performance of the proposed blocks

\begin{tabular}{|l|c|c|}
\hline Virtex XCV 300 & 1st Arch. & IFFT (Optimized) \\
\hline Number of Slices & $73 \%$ & $51 \%$ \\
\hline Number of Flip-Flops & $16 \%$ & $9 \%$ \\
\hline Number of 4-input LUTs & $66 \%$ & $47 \%$ \\
\hline Number of bonded IOBs & $20 \%$ & $19 \%$ \\
\hline Number of BRAMs & $12 \%$ & - \\
\hline Number of GCLKs & $50 \%$ & $25 \%$ \\
\hline Max. Frequency (MHz) & 53 & 55.86 \\
\hline
\end{tabular}

of approximately $20 \%$ of the required arithmetic operations, for the operation of the convolution is achieved for a 802.11 WLAN implementation. The implementation is better than previous designs in terms of computational complexity and requires less chip area.

\section{REFERENCES}

[1] Specification of baseband architecture and implementation complexity. Technical Report IST-1999-10025, WIND-FLEX, 1999.

[2] Wireless LAN MAC and PHY specifications High-Speed Physical Layer in the $5 \mathrm{ghz}$ Band. Technical Report IEEE Std. 802.11a-1999, IEEE, 2000.

[3] M. Faulkner. The effect of filtering on the performance of OFDM systems. IEEE Transactions on Vehicular Technology, 49(5):1877-1884, Sept. 2000.

[4] E. Fotopoulou, V. Paliouras, and T. Stouraitis. A computational technique and a VLSI architecture for digital pulse shaping in OFDM modems. In Proceedings of the 2003 IEEE Symposium on Circuits and Systems (ISCAS 2003), volume 2, pages 125-128, May 2003.

[5] S. He and M. Torkelson. Design and implementation of a 1024-point pipeline FFT processor. In Proceedings of the IEEE Custom Integrated Circuits Conference, pages 131-134, May 1998.

[6] S. Leyonhjelm and M. Faulkner. Comparison of three interpolator designs for use in OFDM transmitters. Electronics Letters, 42(5):289- 291, Mar. 2006. 
[7] A. V. Oppenheim and R. W. Schafer. Discrete-Time Signal Processing. Prentice Hall Inc., 1999.

[8] R. Van Nee and R. Prasad. OFDM Wireless Multimedia Communications. Artech House Publishers, 2000. 